\title{
Organic Composomes as Supramolecular Aptamers
}

\author{
Tracey N. Bell, Keke Feng, Gabriel Calvin, David H. Van Winkle, and Steven Lenhert*
}

Cite This: ACS Omega 2020, 5, 27393-27400

Read Online

ABSTRACT: Information contained in the sequences of biological polymers such as DNA and protein is crucial to determining their function. Lipids are not generally thought of as informationcontaining molecules. However, from a supramolecular perspective, the number of possible combinations of lipids in a mixture is comparable to the complexity of DNA or proteins. Here, we test the idea that an organic composome can exhibit molecular recognition. We use water/octanol as a model two-phase system and investigate the effect of organic solutes in different combinations in the organic phase on selective partitioning of two water-soluble dyes (Brilliant Blue FCF and Allura Red AC)

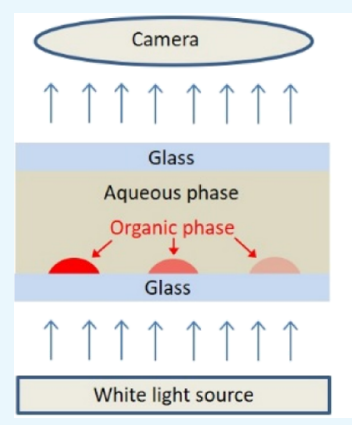

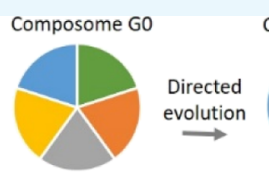

Composome G6

White light source

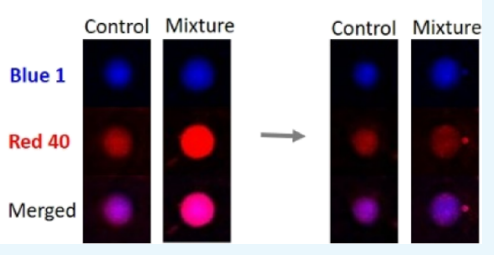
from the aqueous phase into the organic phase. We found that variation in the concentration of the surfactant cetyltrimethylamonium bromide (CTAB) in the octanol phase alone was sufficient to cause a switch in selectivity, with low $\mathrm{CTAB}$ concentrations being selective for the red dye and high CTAB concentrations being selective for the blue dye. Other organic components were added to the organic phase to introduce molecular diversity into the composome and directed evolution was used to optimize the relative concentrations of the solutes. An improvement of selective partitioning in the heterogeneous system over the pure CTAB solution was observed. The results indicate that supramolecular composomes are sufficient for molecular recognition processes in a way analogous to nucleic acid aptamers.

\section{INTRODUCTION}

The modern molecular understanding of biology is based on the idea that the information contained in the sequence of a polymer such as a protein is related to a functional property of that polymer. With 20 amino acids linked together in a linear chain of an average of about 400 residues, there are approximately $20^{400}$ possible proteins. Natural selection samples these permutations by testing individual sequences for specific functions. An appreciation of the relationship between biopolymer sequence and its molecular function has enabled several different types of new bioinspired polymers. For instance, directed evolution of synthetic protein sequences has led to a variety of semisynthetic proteins with engineered properties such as fluorescence, ${ }^{1}$ catalytic activity, ${ }^{2}$ and molecular recognition. ${ }^{3,4}$ Biomimetic polymers such as peptoids have been synthesized and screened through combinatorial methods in order to identify sequences that can selectively bind analytes. ${ }^{5}$ Synthetic nucleic acid sequences that selectively bind targets have been termed aptamers, from Latin roots for parts that fit. ${ }^{6,7}$ In the case of nucleic acid aptamers, amplification of the sequence information that leads to the desired function can be obtained using the polymerase chain reaction. $^{6-8}$

Lipids are not generally thought of as informationcontaining biological molecules. However, consider the number of combinations made possible by mixing different lipids together at different concentrations, which depends on the number of different compounds in the mixture and the precision with which their concentrations are controlled. For instance, if the concentrations of each lipid in the mixture can be varied in increments of 5\% (5 parts per hundred) of the total mass of the mixture, then there would be $100 / 5$ or 20 parts of $5 \%$ being chosen from the set of available lipids that make up the mixture. If we therefore define $(x)$ as the number of parts that make up the mixture in parts-per notation, chosen from $(n)$ different available lipids, then we can calculate the number of possible mixtures using the formula for combinations with repetition

$$
C_{x}^{n+x-1}=\frac{(n+x-1) !}{x !(n-1) !}
$$

Biological lipids are known to be highly heterogeneous, consisting of mixtures of at least 250 chemical species that can reasonably be expected to be maintained at molar or mass fractions between 1 and 5\%, corresponding to values of $x$ from 20 to $100 .^{9-12}$ Considering these parameters, the number of

Received: August 7, 2020

Accepted: September 29, 2020

Published: October 14, 2020 
possible mixtures is on the order of $10^{30}$ to $10^{90}$, which scales comparably to the molecular diversity made possible by DNA and proteins, as plotted in Figure 1.

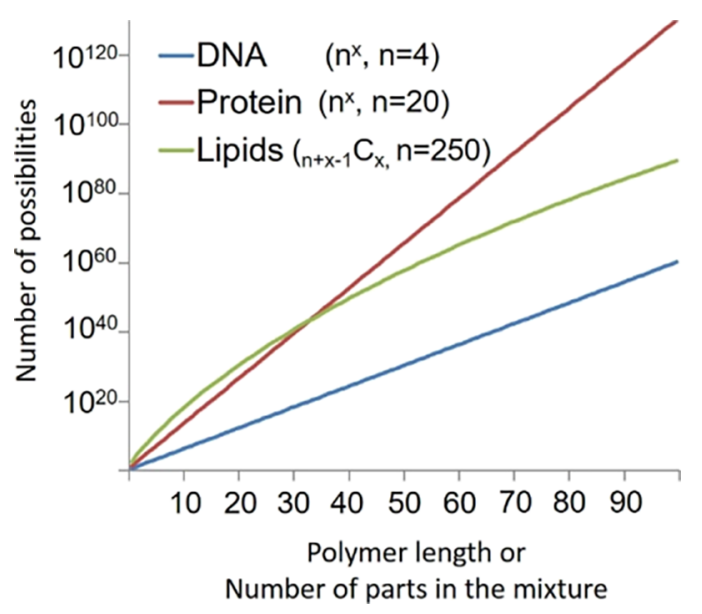

Figure 1. Number of combinations made possible by mixing different lipids together at different combinations, compared to the permutation spaces of biopolymers such as DNA and protein. DNA scales as $4^{x}$ while protein scales as $20^{x}$. Lipid combination space scales with the number of parts that make up the mixture $(x)$ according to formula 1 .

The combinatorial materials field has emerged as a new way of thinking about material properties in terms of a combination space of elemental or molecular components. ${ }^{13}$ This approach has been widely explored for inorganic materials, and applications in organic and biological systems have been considered. ${ }^{14-16}$ From a biological perspective, the idea that an organic "composome" may carry out biological functions is the basis of the lipid world hypothesis for the origin of life. ${ }^{17,18}$ Experimentally, the possibility to evolve unique droplet behaviors reminiscent of cellular behaviors has been demonstrated. ${ }^{19,20}$ Sato et al. have recently used a new in vivo selection approach to identify lipid combinations capable of delivering nucleic acids to specific tissues in animals. ${ }^{21}$ Importantly, in that study, slight differences in relative amounts of lipids in the mixtures were found to be the determining factor for the biological activity.

A supramolecular perspective on lipid structure-function relationships leads to the following question-can specific lipid combinations exhibit properties such as selective binding generally attributed to biological polymers such as proteins or nucleic acids? A traditional structure-function relationship strategy is challenging for addressing this question as the supramolecular lipid structures cannot be purified and characterized as is commonly done with covalent biological polymers. We therefore take a bottom-up synthetic biology approach, where we seek to identify the function in a model system. As a proof of concept, we chose to investigate the ability for the relative organic composition of a mixture to determine selective partitioning of water-soluble dyes into organic phase droplets. Our idea is analogous to the method used to identify nucleic acid-based aptamers, ${ }^{6,7}$ where instead of identifying a polymer sequence that shows selective binding, we vary the composition of the organic phase droplets in order to identify selective partitioning.

\section{RESULTS AND DISCUSSION}

Our experimental setup is illustrated in Scheme 1. The apparatus includes a light source, a color camera, and a

Scheme 1. Microfluidic Apparatus and Chemical Structures Used; (a) Microfluidic Device Containing Adherent Organic Droplets Exposed to Aqueous Dye Solutions While Optical Transmittance Is Monitored Using a Color Camera; (b) CTAB Is Dissolved in the Organic Phase; (c) Blue 1 and Red 40 Dyes Are Dissolved in the Aqueous Phase
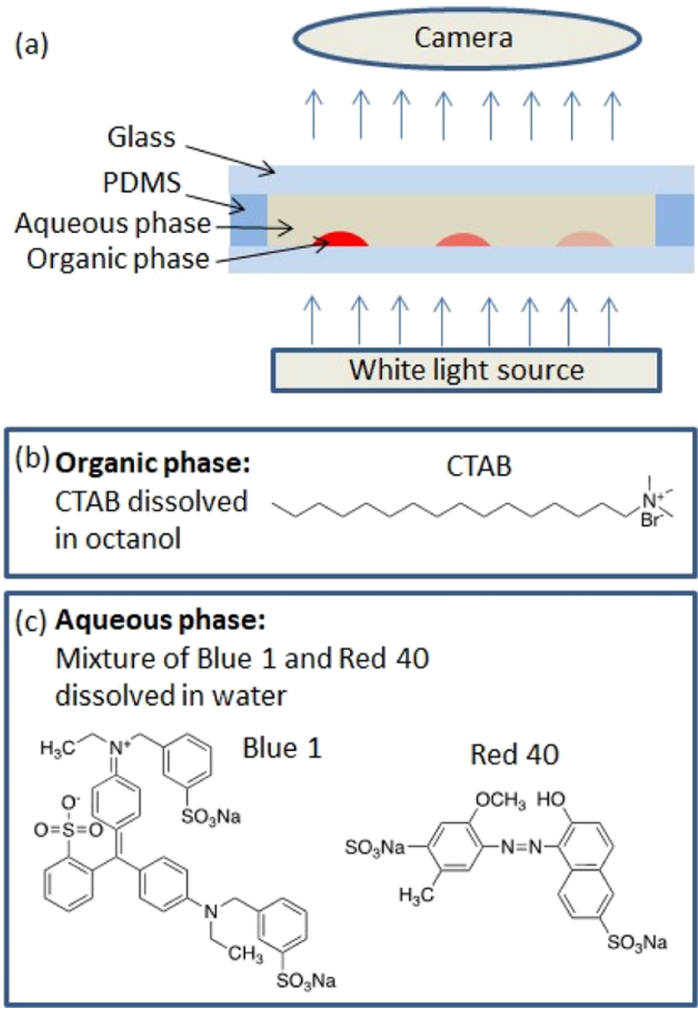

microfluidic flow cell made of glass slides separated by polydimethylsiloxane (PDMS) barriers (Scheme 1a). Octanol was chosen as the organic phase for this study as partitioning between water and octanol is a standard model for understanding the physicochemical properties of biologically relevant small molecules. ${ }^{22}$ Droplets of octanol containing the cationic surfactant cetyltrimethylamonium bromide (CTAB) are deposited in the flow cell and exposed to aqueous solutions of the organic dyes Brilliant Blue FCF (Blue 1) and Allura Red AC (Red 40) as indicators of partitioning. Chemical structures of $\mathrm{CTAB}$ and the dyes are shown in Scheme $1 b, c$. These dyes were selected as they are watersoluble ${ }^{23,24}$ and should have a net charge of -2 in water over a wide $\mathrm{pH}$ range because of the presence of sulfonate groups which have negative $\mathrm{p} K_{\mathrm{a}}$ values. ${ }^{25}$ Furthermore, their optical absorbance values are such that the absorbance can be detected by the red and blue channels of an RGB camera (Figure S1). Calibration curves of such a camera are shown in Figure S2, with tests for crosstalk indicating suitability of these cameras for semiquantitative detection of Blue 1 and Red 40 at concentrations of $10-400 \mu \mathrm{M}$ of Blue 1 and $25-800 \mu \mathrm{M}$ of Red 40 , both in the presence or absence of the other dye.

We observed that selective partitioning for either Red 40 or Blue 1 depends on the $C T A B$ concentration in the organic 

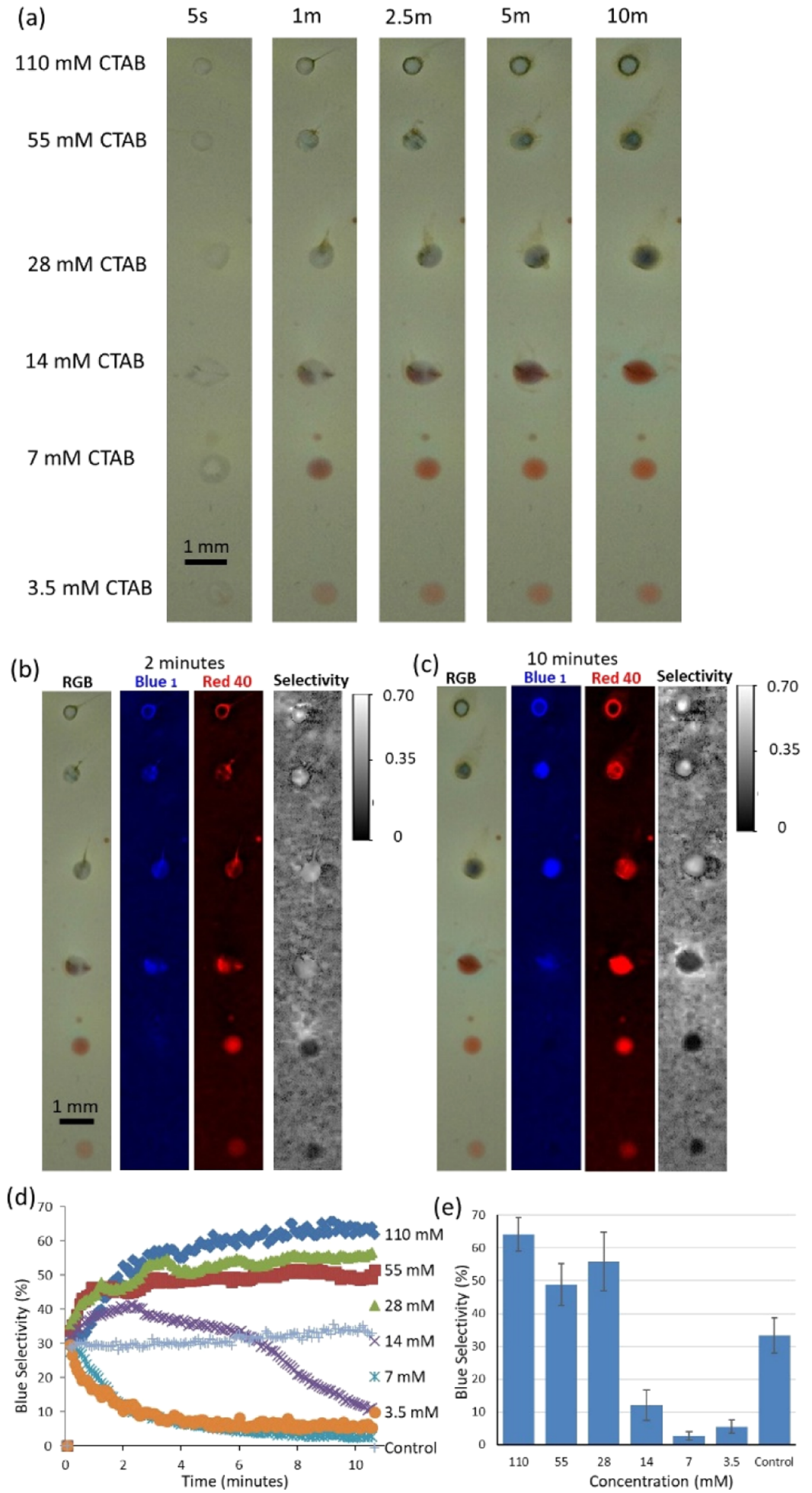

Figure 2. Partitioning selectivity can be controlled by relative composition in a simple binary solution. (a) Octanol droplets containing CTAB monitored by an RGB camera over time show selective partitioning of red or blue dye into the octanol phase depending on the CTAB concentration. (b,c) Processed RGB images at 2 and 10 min show the absorbance due to Blue 1 (red absorbance) and the absorbance due to red 1 (blue absorbance), which are used to calculate selectivity. (d) Graph showing the kinetics of the selective partitioning. (e) Plot of the selectivity measured at $10 \mathrm{~min}$ as a function of CTAB concentration shows a sharp switch in selectivity between 14 and $28 \mathrm{mM}$.

phase, as shown in Figure 2 and the Supporting Information Video. The organic phase appears clear when drops are created. As soon as the aqueous solution is added, the dyes start to partition into the droplets as seen in the first panel of 2a. Droplets with high CTAB concentrations (i.e., 28, 55, and
$110 \mathrm{mM}$ ) started to turn blue within the first minute and continued to increase in blue color throughout the remainder of the sampling period, while samples with low CTAB concentrations (7 and $3.5 \mathrm{mM}$ ) turned red (Figure 2a). Interestingly, samples with an intermediate $\mathrm{CTAB}$ concen- 
tration of $14 \mathrm{mM}$ turned blue within the first minute and then later began to lose blue color and increase in red color throughout the remainder of the sampling period. We found these trends to be qualitatively very reproducible. As the colors observed are based on absorbance, we do not expect a quenching behavior. However, to test for the possibility that $\mathrm{CTAB}$-dependent aggregation may change the optical properties of the dyes, we scaled up the extraction of dyes at $55 \mathrm{mM}$ $\mathrm{CTAB}$ (blue solution) and $7 \mathrm{mM} \mathrm{CTAB} \mathrm{(red} \mathrm{solution)} \mathrm{and}$ serially diluted them in octanol where we observed a linear decrease in both dye's absorbance values with dilution within the sensitive range of the camera (Figure S3). This is consistent with selective partitioning rather than a CTABdependent change in the extinction coefficients of the dyes.

Figure $2 \mathrm{~b}, \mathrm{c}$ shows a more detailed colorimetric analysis of the data at 2 and $10 \mathrm{~min}$, respectively. We used the red and blue channels of an RGB microscope for a semiquantitative analysis of absorbance values for each dye and falsely colored the images to indicate the relative abundance of the dyes. We further used the pixel values in the images to calculate selectivity based on the absorbance calculated for each pixel in the image to produce an image showing selectivity. We define selectivity for the blue dye as $A_{\text {red }} /\left(A_{\text {red }}+A_{\text {blue }}\right)$, where $\left(A_{\text {red }}\right)$ is red absorbance and ( $\left.A_{\text {blue }}\right)$ is blue absorbance, as these values are proportional to the relative dye concentrations within the sensitive range of the camera (Figure S2). The intermediate $\mathrm{CTAB}$ concentration of $14 \mathrm{mM}$ can be seen to switch selectivity over the timescale of this experiment. After $10 \mathrm{~min}$, the blue dye can be seen to be released from the octanol droplets, resulting in a higher apparent blue selectivity in the aqueous phase surrounding these droplets (Figure 2c). No detectable partitioning was observed in pure octanol droplets, which showed the same selectivity as areas in the images between droplets. This experiment demonstrates that selective partitioning can be determined simply by the concentration of a single component in a minimal composome.

The selectivity images in Figure $2 \mathrm{~b}, \mathrm{c}$ show high blue selectivity for the high CTAB concentrations and low blue selectivity for the low CTAB concentrations. The selectivity values are plotted in Figure 2 d,e. From Figure 2 d, it can be seen that the switch in selectivity relative to the background occurs at around $6 \mathrm{~min}$. We noticed that the precise timing of this switch in selectivity appeared to be sensitive to drop size and flow, with smaller droplets switching faster and more flow increasing the switching time. While Figure 2 shows that dyes partitioned into droplets of around $0.5 \mathrm{~mm}$ diameter on timescales of minutes, larger drops with diameters around 2 $\mathrm{mm}$ that touched both sides of the flow cell took hours to show the same trends (Figure S4). Flowing extra dye solution through the chamber was found to speed up the partitioning times, presumably by decreasing boundary layer resistance. In the case of the dye concentrations that switch from blue to red selectivity, the droplets that were closer to the side of the flow cell where the dye was added consistently switched from blue to red first, as they experienced more total flow than the drops near the end of the chamber (Figure S5). We also noticed that slight variations in the dye concentrations in the aqueous phase (e.g., 6.4:1 v/s 8:1 Red 40/ Blue 1 molar ratios) affected the magnitude of the switch at $14 \mathrm{mM} \mathrm{CTAB}$, although the general trend for the other $\mathrm{CTAB}$ concentrations was not affected. $\mathrm{CTAB}$ drops left in water overnight prior to dye exposure showed no qualitative difference in their partitioning behaviors (Figure S6).
These results suggest thinking about the CTAB-dependent switch in selective partitioning from a supramolecular perspective. For instance, the CTAB/octanol/water system has been shown to form several mesophases depending on the molar ratios of the three components, including an isotropic reverse micellar phase at lower $\mathrm{CTAB}$ and water concentrations and a lamellar phase at higher relative CTAB concentrations. $^{26}$ We hypothesize that the sharp CTAB concentration-dependent switch in selectivity (Figure 2e) may result from structural changes in $\mathrm{CTAB}$ reverse micelles in the octanol phase at concentrations above $14 \mathrm{mM}^{26,27}$ From a thermodynamic perspective, reverse micelles at concentrations above $14 \mathrm{mM}$ may exist in a supramolecular structure that selectively binds the blue dye, while aggregates of $\mathrm{CTAB}$ at lower concentrations bind the red dye. As is the case for proteins, such a binding is likely due to an induced fit mechanism, ${ }^{28}$ where the presence of the dye changes the molecular conformations of the aggregated CTAB molecules resulting in an overall lower energy state. Such changes in liquid crystalline, nanoparticulate, and micellar structures have been investigated in drug delivery, allowing the release of encapsulated drugs depending on a change of environment. ${ }^{29}$ From a kinetic perspective, the relative rates of dye crossing the interface may also be modulated by a local supramolecular structure of $\mathrm{CTAB}$ near the interface. The switch in selectivity of the droplets at the intermediate CTAB concentration of 14 $\mathrm{mM}$ suggests that such kinetics may play a role while the system is out of equilibrium, with the blue dye initially partitioning faster, then being replaced by the red dye. This could be due to changes in the electrochemical potential across the interface as ions transfer. ${ }^{30}$ We should also consider the fact that as the blue dye enters the octanol phase and ions exchange across the interface, the composome is once again changing, which may result in new supramolecular structures that favor the red dye partitioning. Investigation into the supramolecular structure-function relationships of these and other organic composomes is a promising area for future research.

To test the idea that specific combinations of heterogeneous solutes might lead to improved selectivity compared to CTAB in octanol only, the experiment shown in Scheme 2 was designed. A chemical alphabet made up of five organic compounds was created, abbreviated with letters A-E (see Scheme 2a). We chose organic compounds containing quaternary amine, primary amine, fatty acid, and alcohol functional groups. CTAB was the only compound that showed selectivity for blue dye by itself when dissolved in octanol (Figure S7). Using the five components, the size of the combination space made possible by mixing different lipids together at different concentrations is shown in Scheme $2 b$, as a function of the number of parts making up the mixture. The composomes were varied using directed evolution according to the process shown in Scheme 2c. We started with an equimolar solution and formed droplet arrays that vary the concentration of a single component using factors of $2 x, 4 x$, and $0.5 x$ and $0.25 x$ for a total of 5 samples per generation with approximately 10 replicates of each composition (Figure S8). Selection of the optimal concentration was based on blue selectivity as defined previously. The optimal concentration for that component was then fixed, and the next component was varied in an iterative process until the selectivity no longer increased. 
Scheme 2. Directed Evolution of a Composome for Selective Partitioning of Blue Dye through Directed Evolution; (a) The Alphabet Shown Is (A) CTAB; (B) Didodecyldimethylamonium Bromide; (C) Stearic Acid; (D) Hexadecane; (E) 1Octadecylamine (b) Calculation of the Number of Combinations Made Possible by Mixing the Different Components Together at Different Concentrations; (c) Illustration of the Directed Evolution Process ${ }^{a}$
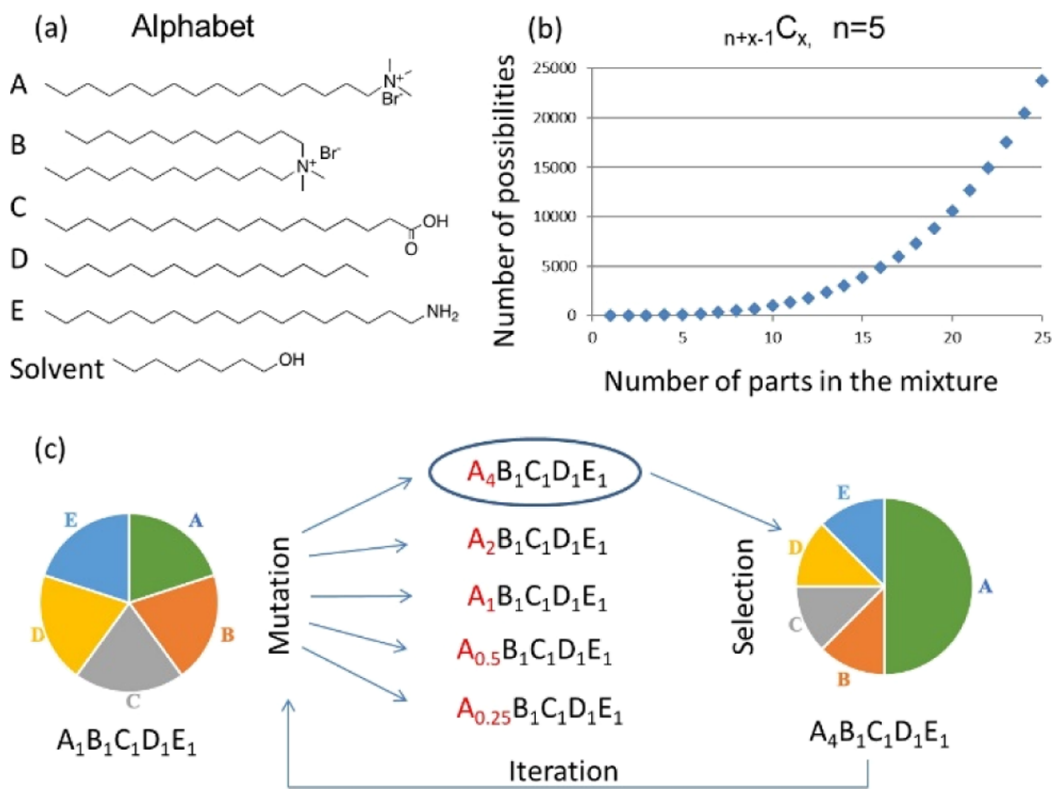

${ }^{a}$ An equimolar initial composome is mutated by varying the concentration of one component at a time followed by a selection process. The subscripts represent the relative concentrations of the mixture.

Figure 3 shows the results of the directed evolution experiment, indicating an improved selectivity compared to control solutions with pure CTAB at $30 \mathrm{mM}$. Although the initial, equimolar mixture was significantly less selective than the control, the selectivity improved significantly after the second generation and remained $20 \%$ more selective than the control. After six generations, the concentration of component A (CTAB) was varied again, but there was no detectable improvement. Our ability to continue to evolve the system was found to be limited by error in quantifying the selectivity. The blue selectivity of composomes evolved by this method, however, were found to be reproducibly improved over the control. Sources of error in these experiments include variations in flow upon addition of the dye, variation in drop size, variations in the optical setups ability to quantify the dye, and possible variation in supramolecular structures formed by mixing procedures, precipitation, and repeated use of stock solutions. We expect reduction in these errors may allow further improvements in selectivity. It is also possible that the system had reached a local maximum in selectivity, suggesting that more sophisticated approaches to sampling the combination space might lead to further improvements.

\section{CONCLUSIONS}

Lipid aggregates form the organic phase of biology and are so far best understood in terms of physical properties such as fluidity, rigidity, membrane tension, and dynamics. ${ }^{32}$ The results here suggest a new way of thinking about lipid biology terms of spatiotemporal organization of functional groups as is commonly done in the case of the protein structure. Although there is an entropic cost involved in the noncovalent aggregation of small molecules compared to covalent polymers, an organic composome is also more likely to form and be degraded through mixing. Such a mechanism therefore has

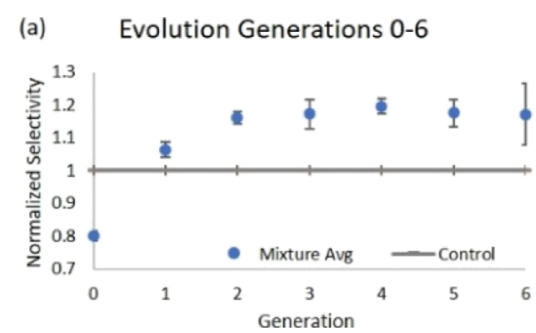

(b)
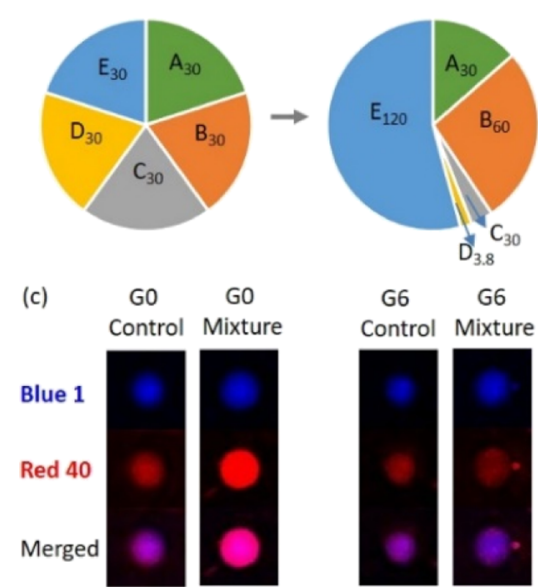

Figure 3. Directed evolution of a lipid composome leading to improved selectivity compared to pure CTAB. (a) Graph comparing the control to the generations and their relative selectivity. (b) Pie charts showing the relative concentrations from the starting generation to the 6th generation. (c) Micrographs showing the absorbance due to the blue dye and due to the red dye for the starting generation $\left(\mathrm{G}_{0}\right)$ and after the 6th generation $\left(\mathrm{G}_{6}\right)$. 
implications for the origin of life. The biological implications of this perspective suggest a supramolecular level of biological regulation by relative lipid compositions in biological membranes, vesicles, lipid droplets, and other nonaqueous aggregates in living systems. ${ }^{12}$ For example, dynamic supramolecular lipid nanostructure-function relationships such as lipid rafts, ${ }^{33}$ membrane curvature, ${ }^{34}$ and fluctuations in multilamellar systems such as myelin ${ }^{35}$ are likely regulated by relative lipid composition. This perspective opens new possibilities in the identification of supramolecular catalysts, ${ }^{36}$ drug targets, ${ }^{37}$ and biosensors. ${ }^{38,39}$

\section{METHODS}

4.1. Organic-Phase Preparation. Organic solutes included cetyltrimethylamonium bromide (CTAB); 1-Octadecylamine (ODA); didodecyldimethylamonium bromide (DDAB); stearic acid (SA); and hexadecane (HDA). ODA (L15458) was purchased from Alfa Aesar Chemical (Ward Hill, MA). CTAB (H5882), SA (85679), DDAB (359025), and 1-octanol (112615) were purchased from Sigma-Aldrich (St. Louis, USA). HDA (H0066) was purchased from Tokyo Chemical Industry (TCI America). Stock solutions were prepared in chloroform and sonicated at room temperature. The solutions were prepared and stored in amber vials for repeated use and reheated as needed prior to use. Mixtures were prepared in PCR tubes, and bulk chloroform was evaporated in a nitrogen stream, followed by drying in a vacuum a minimum of $30 \mathrm{~min}$. 1-Octanol was added to the dried solutes to reach the appropriate concentration, and the solutions were lightly vortexed for $10 \mathrm{~s}$, heated in a water bath at $60{ }^{\circ} \mathrm{C}$ for $10 \mathrm{~min}$, and then sonicated for $10 \mathrm{~min}$. The solutions were stored for repeated use; after storage, sonication, heating, and vortexing were repeated as needed to achieve transparent solutions prior to use.

4.2. Aqueous-Phase Preparation. Erioglaucine disodium salt (Blue 1) was purchased from Sigma-Aldrich (St. Louis, MO, USA). Allura red AC (Red 40) was obtained from TCI (Tokyo, Japan). The dyes were dissolved in deionized water at various concentrations for calibration of the optical microscope. Mixtures of the dye solutions for the experiment shown in Figure 2 were prepared at $0.20 \mathrm{~g} / \mathrm{L}(403 \mu \mathrm{M})$ for Red 40 and $0.05 \mathrm{~g} / \mathrm{L}(63 \mu \mathrm{M})$ for Blue 1 . For the directed evolution experiments, a mixture of the dyes was prepared with the blue dye at $50 \mu \mathrm{M}$ and the red dye at $400 \mu \mathrm{M}$. These concentrations were found to result in values within the detectable range of the optical microscope used to measure absorbance.

4.3. Microarraying and Flow Cell Assembly. The different lipid solutions were microarrayed using $0.1-10 \mu \mathrm{L}$ disposable sterile pipet tips (VWR) directly onto the substrate. After tapping the microarray onto the substrate, $0.3 \mathrm{~mm}$ thick PDMS or $0.1 \mathrm{~mm}$ thick double-side tape was used as a spacer between the substrate and the cover. The two spacers were fixed $1.8 \mathrm{~cm}$ apart onto the substrate. The coverslip was placed carefully onto two spacers. Microscope slides manufactured by Azer Scientific (Unifrost) were used as substrates. Microscope cover glasses $(25 \mathrm{~mm} \times 25 \mathrm{~mm})$ manufactured by VWR were used as slip covers. Approximately $130 \mu \mathrm{L}$ of dye solution was added to the right side of the flow cell moved quickly through the flow cell. For the directed evolution experiments, samples were washed with $1 \mathrm{~mL}$ of deionized water prior to imaging.

4.4. Optical Characterization. Samples requiring $30 \mathrm{~min}$ or less were imaged with a digital microscope (Dino-Lite Pro2
AD-413T or Dino-Lite Edge AM4115ZT) at 100× magnification. A picture was taken before and after addition of the coverslip to determine if drops touched both glass surfaces. For imaging lipid microarrays, a portable back light stage (DinoLite MSBL-ZW1R) was used as a white light source and positioned under the bottom of the slide to illuminate the sample. Collection of optical absorption spectra of Red 40 and Blue 1 was carried out using a $0.5 \mathrm{~cm} \times 0.5 \mathrm{~cm}$ quartz cuvette on a UV 2450 UV-vis absorption spectrophotometer (Shimadzu, Columbia, MD). Images for measurement of the absorbance were captured in brightfield mode. The timedependent partitioning was recorded at one frame per second interval. Samples requiring more than $30 \mathrm{~min}$ (Figure S4) imaging time were prepared as follows. The slide chamber was placed in a Petri dish containing a damp tissue to maintain a humid atmosphere throughout imaging and sealed with Parafilm. For imaging lipid microarrays in the slide chamber, RGB images were captured with a Raspberry Pi using Camera Module V2-8 Megapixel, 1080p with an LED adjustable illuminated tracing light box (GGE Corp., Limited, China) positioned under the bottom of Petri dish to illuminate the sample.

4.5. Data Analysis. Absorbance values were calculated by separating the images in to RGB channels. As the red dye has an absorbance maximum in the blue, and the blue dye has an absorbance maximum in the red, the gray values of the red and blue channels were used to calculate absorbance (Figure S1). As a blank, an image was taken without any dye. Blue selectivity was calculating using red absorbance $\left(A_{\text {red }}\right)$ and blue absorbance $\left(A_{\text {blue }}\right)$ values according to $A_{\text {red }} /\left(A_{\text {red }}+A_{\text {blue }}\right)$. The optical microscope was calibrated using varying concentrations of dyes (Figure S2), and the dye concentrations were chosen to be visible within the dynamic range of the camera. Pixel intensities were extracted using image processing software, ImageJ (downloaded from the NIH website, http://imagej.nih. gov/ij/). Analysis was automated by the use of a script written for the statistical computation program "R." The software used coordinates extracted from the image processing program (ImageJ) and created a macro line item for circles of diameter 6 pixels (unless otherwise noted) centered at the coordinates given. The " $R$ " script then acquired the pixel values from the red and blue channels and used them to calculate absorbance and selectivity values.

4.6. Directed Evolution. Selectivity values at $10 \mathrm{~min}$ were measured and used to identify the compositions with the highest blue selectivity. Drops were discarded from evaluation if there was significant movement (more than $1 \mathrm{~mm}$ from the original placement) or if the drop was not completely under the coverslip. If a drop appeared to separate out into multiple smaller drops, the smaller drops were discarded from the analysis. In cases where there is a tie (selection is within two standard deviations of the selectivity value), the following criteria were used to choose the optimal sample. The droplets must have been in the detectable range for absorbance. The selection of less broken drops was chosen such that final drop size was at least $3 / 4$ of its original size.

\section{ASSOCIATED CONTENT}

\section{Supporting Information}

The Supporting Information is available free of charge at https://pubs.acs.org/doi/10.1021/acsomega.0c03799. 
Materials and methods and size/shape partitioning kinetics calibration curves (PDF)

Selective partitioning for Red 40 and Blue 1 depending on the CTAB concentration in the organic phase (AVI)

\section{AUTHOR INFORMATION}

\section{Corresponding Author}

Steven Lenhert - Department of Biological Science and Integrative NanoScience Institute, Florida State University, Tallahassee, Florida 32306, United States; @ orcid.org/00000002-2749-4229; Email: lenhert@bio.fsu.edu

\section{Authors}

Tracey N. Bell - Department of Biological Science and Integrative NanoScience Institute, Florida State University, Tallahassee, Florida 32306, United States

Keke Feng - Department of Physics, Florida State University, Tallahassee, Florida 32306, United States

Gabriel Calvin - Department of Biological Science and Integrative NanoScience Institute, Florida State University, Tallahassee, Florida 32306, United States

David H. Van Winkle - Department of Physics, Florida State University, Tallahassee, Florida 32306, United States

Complete contact information is available at:

https://pubs.acs.org/10.1021/acsomega.0c03799

\section{Author Contributions}

Experiments were carried out by T.N.B., K.F., and S.L. under the direction of D.H.V.W. and S.L. G.C. wrote the R script used for data analysis. S.L. conceived of the study and wrote the manuscript. All authors have given approval to the final version of the manuscript.

\section{Notes}

The authors declare no competing financial interest.

\section{ACKNOWLEDGMENTS}

The authors thank Drs. Lei Zhu, Scott Stagg, Jonathan Dennis, Jingiiao Guan, and Mike Roper for the helpful discussions, and Dr. Hedi Mattoussi for help with spectrophotemetry. S.L. thanks Riona Westphal and other members of the FSU Young Scholars Program for assistance with initial experiments.

\section{ABBREVIATIONS}

RGB, red green blue; $C T A B$, cetyltrimethylamonium

\section{REFERENCES}

(1) Shaner, N. C.; Campbell, R. E.; Steinbach, P. A.; Giepmans, B. N. G.; Palmer, A. E.; Tsien, R. Y. Improved monomeric red, orange and yellow fluorescent proteins derived from Discosoma sp red fluorescent protein. Nat. Biotechnol. 2004, 22, 1567-1572.

(2) Sheldon, R. A.; Brady, D. The limits to biocatalysis: pushing the envelope. Chem. Commun. 2018, 54, 6088-6104.

(3) Hoogenboom, H. R. Selecting and screening recombinant antibody libraries. Nat. Biotechnol. 2005, 23, 1105-1116.

(4) Smith, G. Filamentous Fusion Phage - Novel Expression Vectors That Display Cloned Antigens on the Virion Surface. Science 1985, 228, 1315-1317.

(5) Kim, J. H.; Kim, S. C.; Kline, M. A.; Grzincic, E. M.; Tresca, B. W.; Cardiel, J.; Karbaschi, M.; Dehigaspitiya, D. C.; Chen, Y.; Udumula, V.; Jian, T.; Murray, D. J.; Yun, L.; Connolly, M. D.; Liu, J.; Ren, G.; Chen, C.-L.; Kirshenbaum, K.; Abate, A. R.; Zuckermann, R. N. Discovery of Stable and Selective Antibody Mimetics from Combinatorial Libraries of Polyvalent, Loop-Functionalized Peptoid Nanosheets. ACS Nano 2020, 14, 185-195.
(6) Tuerk, C.; Gold, L. Systematic evolution of ligands by exponential enrichment: RNA ligands to bacteriophage T4 DNA polymerase. Science 1990, 249, 505-510.

(7) Ellington, A. D.; Szostak, J. W. In vitro selection of RNA molecules that bind specific ligands. Nature 1990, 346, 818-822.

(8) Saiki, R.; Scharf, S.; Faloona, F.; Mullis, K.; Horn, G.; Erlich, H.; Arnheim, N. Enzymatic amplification of beta-globin genomic sequences and restriction site analysis for diagnosis of sickle cell anemia. Science 1985, 230, 1350-1354.

(9) Wenk, M. R. The emerging field of lipidomics. Nat. Rev. Drug Discov. 2005, 4, 594-610.

(10) van Meer, G.; Voelker, D. R.; Feigenson, G. W. Membrane lipids: where they are and how they behave. Nat. Rev. Mol. Cell Biol. 2008, 9, 112-124.

(11) He, H.; Emmett, M. R.; Nilsson, C. L.; Conrad, C. A.; Marshall, A. G. High mass accuracy and resolution facilitate identification of glycosphingolipids and phospholipids. Int. J. Mass Spectrom. 2011, 305, 116-119.

(12) Harayama, T.; Riezman, H. Understanding the diversity of membrane lipid composition. Nat. Rev. Mol. Cell Biol. 2018, 19, 281296.

(13) Xiang, X.-D.; Sun, X.; Briceno, G.; Lou, Y.; Wang, K.-A.; Chang, H.; Wallace-Freedman, W. G.; Chen, S.-W.; Schultz, P. G. A combinatorial approach to materials discovery. Science 1995, 268, $1738-1740$.

(14) Chen, P.-C.; Liu, X.; Hedrick, J. L.; Xie, Z.; Wang, S.; Lin, Q.Y.; Hersam, M. C.; Dravid, V. P.; Mirkin, C. A. Polyelemental nanoparticle libraries. Science 2016, 352, 1565-1569.

(15) Bawazer, L. A.; McNally, C. S.; Empson, C. J.; Marchant, W. J.; Comyn, T. P.; Niu, X.; Cho, S.; McPherson, M. J.; Binks, B. P.; deMello, A.; Meldrum, F. C. Combinatorial microfluidic droplet engineering for biomimetic material synthesis. Sci. $A d v$. 2016, 2, No. e1600567.

(16) Kroiss, D.; Ashkenasy, G.; Braunschweig, A. B.; Tuttle, T.; Ulijn, R. V. Catalyst: can systems chemistry unravel the mysteries of the chemical origins of life? Chem 2019, 5, 1917-1920.

(17) Lancet, D.; Zidovetzki, R.; Markovitch, O. Systems protobiology: origin of life in lipid catalytic networks. J. R. Soc. Interface 2018, 15, 20180159.

(18) Segré, D.; Ben-Eli, D.; Deamer, D. W.; Lancet, D. The lipid world. Orig. Life Evol. Biosph. 2001, 31, 119-145.

(19) Doran, D.; Rodriguez-Garcia, M.; Turk-MacLeod, R.; Cooper, G. J. T.; Cronin, L. A recursive microfluidic platform to explore the emergence of chemical evolution. Beilstein J. Org. Chem. 2017, 13, $1702-1709$.

(20) Gutierrez, J. M. P.; Hinkley, T.; Taylor, J. W.; Yanev, K.; Cronin, L. Evolution of oil droplets in a chemorobotic platform. Nat. Commun. 2014, 5, 5571.

(21) Sago, C. D.; Lokugamage, M. P.; Islam, F. Z.; Krupczak, B. R.; Sato, M.; Dahlman, J. E. Nanoparticles that deliver RNA to bone marrow identified by in vivo directed evolution. J. Am. Chem. Soc. 2018, 140, 17095-17105.

(22) Lipinski, C. A.; Lombardo, F.; Dominy, B. W.; Feeney, P. J. Experimental and computational approaches to estimate solubility and permeability in drug discovery and development settings. Adv. Drug Delivery Rev. 1997, 23, 3-25.

(23) National Center for Biotechnology Information. PubChem Compound Summary for CID 33259, Allura Red AC Dye, 2020.

(24) National Center for Biotechnology Information. PubChem Compound Summary for CID 19700, Brilliant Blue FCF, 2020.

(25) Smith, M.; March, J. March's Advanced Organic Chemistry: Reactions, Mechanisms, and Structure, 6th ed.; Wiley-Interscience: Hoboken, N.J., 2007.

(26) Fontell, K.; Khan, A.; Lindström, B.; Maciejewska, D.; PuangNgern, S. Phase-Equilibria and Structures in Ternary-Systems of a Cationic Surfactant (C16tabr or (C16ta)2so4), Alcohol, and Water. Colloid Polym. Sci. 1991, 269, 727-742.

(27) Ueda, A. C.; de Oliveira, L. H.; Hioka, N.; Aznar, M. LiquidLiquid Extraction of Basic Yellow 28, Basic Blue 41, and Basic Red 46 
Dyes from Aqueous Solutions with Reverse Micelles. J. Chem. Eng.

Data 2011, 56, 652-657.

(28) Koshland, D. E. Application of a Theory of Enzyme Specificity to Protein Synthesis. Proc. Natl. Acad. Sci. U.S.A. 1958, 44, 98-104.

(29) Aleandri, S.; Mezzenga, R. The physics of lipidic mesophase delivery systems. Phys. Today 2020, 73, 38-44.

(30) Marcus, R. A. On the theory of ion transfer rates across the interface of two immiscible liquids. J. Chem. Phys. 2000, 113, 16181629.

(31) Brown, K. A.; Brittman, S.; Maccaferri, N.; Jariwala, D.; Celano, U. Machine Learning in Nanoscience: Big Data at Small Scales. Nano Lett. 2020, 20, 2-10.

(32) Ho, J. C. S.; Rangamani, P.; Liedberg, B.; Parikh, A. N. Mixing Water, Transducing Energy, and Shaping Membranes: Autonomously Self-Regulating Giant Vesicles. Langmuir 2016, 32, 2151-2163.

(33) Tayebi, L.; Ma, Y.; Vashaee, D.; Chen, G.; Sinha, S. K.; Parikh, A. N. Long-range interlayer alignment of intralayer domains in stacked lipid bilayers. Nat. Mater. 2012, 11, 1074-1080.

(34) Lowry, T. W.; Hariri, H.; Prommapan, P.; Kusi-Appiah, A.; Vafai, N.; Bienkiewicz, E. A.; Van Winkle, D. H.; Stagg, S. M.; Lenhert, S. Quantification of Protein-Induced Membrane Remodeling Kinetics In Vitro with Lipid Multilayer Gratings. Small 2016, 12, $506-515$.

(35) Di Gioacchino, M.; Bianconi, A.; Burghammer, M.; Ciasca, G.; Bruni, F.; Campi, G. Myelin basic protein dynamics from out-ofequilibrium functional state to degraded state in myelin. Biochim. Biophys. Acta, Biomembr. 2020, 1862, 183256.

(36) Mosca, S.; Ajami, D.; Rebek, J. Recognition and sequestration of $\omega$-fatty acids by a cavitand receptor. Proc. Natl. Acad. Sci. U.S.A. 2015, 112, 11181-11186.

(37) Oprea, T. I.; Bologa, C. G.; Brunak, S.; Campbell, A.; Gan, G. N.; Gaulton, A.; Gomez, S. M.; Guha, R.; Hersey, A.; Holmes, J.; Jadhav, A.; Jensen, L. J.; Johnson, G. L.; Karlson, A.; Leach, A. R.; Ma'ayan, A.; Malovannaya, A.; Mani, S.; Mathias, S. L.; McManus, M. T.; Meehan, T. F.; von Mering, C.; Muthas, D.; Nguyen, D.-T.; Overington, J. P.; Papadatos, G.; Qin, J.; Reich, C.; Roth, B. L.; Schürer, S. C.; Simeonov, A.; Sklar, L. A.; Southall, N.; Tomita, S.; Tudose, I.; Ursu, O.; Vidović, D.; Waller, A.; Westergaard, D.; Yang, J. J.; Zahoránszky-Köhalmi, G. Unexplored therapeutic opportunities in the human genome. Nat. Rev. Drug Discov. 2018, 17, 317-332.

(38) Lenhert, S.; Brinkmann, F.; Laue, T.; Walheim, S.; Vannahme, C.; Klinkhammer, S.; Xu, M.; Sekula, S.; Mappes, T.; Schimmel, T.; Fuchs, H. Lipid multilayer gratings. Nat. Nanotechnol. 2010, 5, 275279.

(39) Huang, D.; Zhao, T.; Xu, W.; Yang, T.; Cremer, P. S. Sensing Small Molecule Interactions with Lipid Membranes by Local $\mathrm{pH}$ Modulation. Anal. Chem. 2013, 85, 10240-10248. 